\title{
Admission Control and Profitability Analysis in Dynamic Spectrum Access Data Networks
}

\author{
Sinem Kockan \\ Department of Electrical and Computer \\ Engineering \\ Boston University \\ Boston, MA 02215 \\ kockan@bu.edu
}

\author{
David Starobinski \\ Department of Electrical and Computer \\ Engineering \\ Boston University \\ Boston, MA 02215 \\ staro@bu.edu
}

\begin{abstract}
New regulations grant network service providers with the right to lease their spectrum to short-term leased secondary users (SUs) for opportunistic usage. In this work, we tackle the challenge of determining admission control and pricing policies on SUs that guarantee profitability under general secondary demand and general traffic models, and accurately reflect the operation of modern cellular data networks (e.g., LTE) in which resources are shared rather than rigidly partitioned. We first analyze the joint problem of bandwidth allocation and admission control of elastic secondary users. We assume Poisson session arrivals, where each session is composed of arbitrarily distributed, and possibly correlated, on and off periods. Under balanced bandwidth allocation, we show that the steady state distribution of the number of active users in the network is insensitive to traffic characteristics beyond their means. This result holds for arbitrary occupancy-based admission control policies on SUs. Next, we prove that the optimal occupancy-based admission control policy is of threshold type, which means that secondary user arrivals are accepted when the total number of active users in the network is below a certain threshold; otherwise, they are rejected. Finally, we identify a price, referred to as the break-even price, and an admission control policy which, together, ensure profitability for any price greater than the break-even price, irrespective of the shape of the secondary demand function.
\end{abstract}

\section{Keywords}

Elastic traffic, Markov decision processes, Pricing, Processor sharing, Insensitivity.

\section{INTRODUCTION}

Measurement studies show that the spectrum allocated to network providers is not fully utilized. According to the Federal Communications Commission (FCC), temporal and spatial spectrum utilization varies from $15 \%$ to $85 \%$ [1]. A

Permission to make digital or hard copies of all or part of this work for personal or classroom use is granted without fee provided that copies are not made or distributed for profit or commercial advantage and that copies bear this notice and the full citation on the first page. To copy otherwise, to republish, to post on servers or to redistribute to lists, requires prior specific permission and/or a fee.

ValueTools'13, December 10 - 12 2013, Turin, Italy

Copyright 2013 ACM 978-1-4503-2539-4/13/12 ...\$15.00. recent study by Cisco shows that data traffic is expected to increase yearly by $55 \%$ through 2017 [9]. The increasing demand, limited network capacity and inefficiency of current spectrum allocation methods create a need for new approaches to use the available spectrum more efficiently.

Along these lines, Dynamic Spectrum Access (DSA) networks have been proposed to solve the problem of inefficient spectrum utilization [1]. DSA networks allow opportunistic users to access licensed bands without interfering with long-term contracted users. The FCC took a major step to facilitate DSA networks by publishing the so-called Report and Order and Further Notice of Proposed Rulemaking [10]. This report defines new policies that support secondary spectrum markets and facilitate opportunistic access to the wireless spectrum through the use of spectrum leasing.

In this work, we consider a model under this realm consisting of two type of customers: Primary Users (PU) and Secondary Users (SU). PUs have a long-term lease, while SUs have a short-term lease with the service provider. To manage spectrum leasing, we consider applying occupancybased admission control on SUs to control their access to the shared network. These policies admit or reject SUs based on the total number of active users in the network at the time of SU arrivals.

The majority of previous work on DSA networks consider admission control and pricing of voice and streaming traffic in loss networks $[3,14,23,25]$. In such networks, bandwidth is partitioned into channels with fixed capacity, thus limiting the maximum allowable number of users in the network to the total number of channels and fixing the bandwidth allocated to each user. In contrast, in our work, we consider elastic traffic models, that capture various TCP-based applications, such as web and file transfer, and rate-adaptive voice and video applications.

In general, data users are limited by a peak bit rate, for instance the access speed to the network. Elastic data traffic users transmit at their peak bit rate if the network is not saturated. However, their rate is adjusted when the network is saturated, in order to prevent congestion. Motivated by the dimensioning of Long Term Evolution (LTE) access networks for elastic traffic [17], we model the network as a processor sharing network for which the bandwidth is not partitioned but rather shared between admitted users according to a bandwidth allocation scheme. In processor sharing networks there is no tight bound on the number of users that can be served simultaneously. Compared to loss networks, processor sharing networks can serve more users 
and answer the need of time-varying bandwidth demands.

Previous measurement studies, such as $[4,5,11,26]$ show that Poisson session arrivals is a realistic assumption to model elastic data traffic. A session is defined as a combination of related flows originated by the same user or application. Thus, sessions can be envisioned as a collection of a fixed or random number of alternating "on" and "off" periods, which lengths follow general probability distributions that may be correlated. An "on" period corresponds to an ongoing flow. At any given time, a user session is considered active if an ongoing flow is present.

The goal of this paper is two-fold: derive traffic-insensitive admission control policies on SUs that grant service providers with maximum average profit, and provide guidance to determine when opening network to spectrum access is profitable when secondary demand is unknown.

Our main contributions are as follows: First,we show that, under a joint occupancy-based admission control policy and balanced bandwidth allocation, the steady state distribution of the number of ongoing flows in the network is insensitive to the traffic characteristics except through their means. Next, we show that the optimal occupancy-based admission control policy of SUs that maximizes the average profit over an infinite time horizon is of threshold type. This policy admits an SU session arrival if and only if the total number of ongoing flows in the network is below a certain threshold. Finally, we analyze the profitability of secondary spectrum leasing under general secondary demand. We prove that there exists a unique price, the so-called break-even price, such that below the break-even price there exists no profitable price to charge the secondary demand. Further, we provide an admission control policy that guarantees profitability for any price exceeding the break-even price.

The rest of the paper is organized as follows: Section 2 presents related work. We describe our network, traffic, and economic models in Section 3. The analysis of the model and characterization of optimal admission control policies are provided in Section 4. We establish profitability conditions of secondary access provisioning in Section 5. Section 6 concludes the paper.

\section{RELATED WORK}

Related work can be classified in three categories: (i) admission control in loss networks with inelastic voice and streaming traffic, (ii) insensitive admission control policies for data traffic in processor sharing networks, and (iii) profitability analysis of DSA loss networks with voice traffic.

In loss networks, bandwidth is partitioned into channels and the number of users that can be served is bounded by the total number of channels. Admission control of delay sensitive traffic in loss networks has been widely studied in the literature. Earlier work includes [18] and [22], which study a profit maximization problem for a multi-class loss network. Recent studies, such as [23], study a call admission control problem to maximize average profit of a service provider in a preemptive DSA loss network. They consider a two-class user network where one class has a higher priority and can preempt the lower priority class traffic when all resources (channels) are fully utilized. Ref. [25] and [14] study profit maximization in multi-class loss networks with streaming traffic. The majority of these works assume Poisson call (flow) arrivals. Different from these works, we analyze a profit maximization problem in a processor sharing network with elastic traffic, and consider a realistic model for data traffic based on Poisson session arrivals.

Next, we survey related work on admission control of elastic traffic in processor sharing networks. We concentrate on the set of work for which the admission control policies are insensitive to traffic characteristics, except through their means. Refs. [16], [21] and [12] study the problem of minimizing metrics related to the blocking probability of users by applying admission control. Ref. [16] considers both streaming and elastic traffic. The authors propose a measurementbased admission control scheduling policy, called Priority Fair Queueuing (PFQ), that admits a new flow if the admitted flow does not reduce the throughput of ongoing elastic flows below a certain threshold. Ref. [21] considers a multiclass network where classes are defined by their minimum rate requirements, and proposes computationally feasible sub-optimal admission control policies. Ref. [12] considers a multi-class network, where each class has a different intensity and derives bounds on the performance of the optimal admission control policy. The above papers focus on multiclass networks, where different classes have different traffic characteristics. As such, finding the structure of the optimal admission control policy is generally difficult. In contrast, in our paper, we consider an economic model where PUs and SUs utilize similar applications and have the same traffic intensity, but differ in their economic values. Further, our metrics of interest focus on the average profit and profitability rather than on the blocking probability. Nevertheless, our results also hold for general data traffic models.

Next, we present related work that establish conditions guaranteeing profitability of secondary spectrum access. Ref. [3] derives the profitability region of a two class DSA loss network with inelastic voice traffic when the relationship between the secondary price (the price charged on SUs) and secondary demand (the arrival rate of SUs subject to a secondary price) is unknown. Both our work and the work in [3] show that the profitability region is insensitive to the secondary demand function and sharing the spectrum with secondary demand can always be made profitable if the secondary price exceeds the so-called break-even price. However, our work is based on considerably different traffic and resource allocation models.

\section{MODEL}

We model the network as a single link processor sharing network with capacity $C$ bits/sec shared by all users in the system and consider elastic data traffic. We model the data traffic at flow and session levels. Previous studies show that a flow, corresponding to a transfer of some digital document like a web-page, is not usually generated in isolation but belongs to a session which consists of other flows belonging to the same application or document created by a user [8]. In general, a session can be described by a collection of "on" and "off" periods, where an "on" period correspond to a flow transmission and an "off" period refers to an interval of inactivity period that separates flows which are generated within a session. The duration of "on" and "off" periods have arbitrary distributions and may be correlated.

We assume that sessions of each PU and SU are i.i.d. and arrive as a Poisson process with rates $\lambda_{1}$ and $\lambda_{2}$ bits/sec respectively. We also assume that each session brings $1 / \mu$ bits on average. The number of flows in each session and the size (in bits) of each flow are arbitrarily distributed, 
beside the constraint on the mean session size. We refer to $\rho_{1}=\lambda_{1} / \mu$ and $\rho_{2}=\lambda_{2} / \mu$ as the intensity of PU and SU sessions, respectively. We assume that $\rho_{1} \leq C$, otherwise PUs would saturate the network even without SUs. We refer to this traffic model as the "Poisson session arrivals" model.

We assume that PUs and SUs share the same peak rate constraint denoted by $R$ bits/sec. We consider elastic traffic where users get bandwidth equal to their peak rate $R$ if the network is not saturated and are required to reduce their bit rate to avoid congestion when the network is saturated. We define $\phi_{1} \geq 0$ and $\phi_{2} \geq 0$ to be the total bandwidth allocated to PU flows and SU flows, respectively. It is assumed that the total bandwidth allocated for a class is equally shared between the flows of that class. For a bandwidth allocation to be feasible, it must satisfy $\phi_{1}+\phi_{2} \leq C$.

Sessions are composed of "on" and "off" periods, which correspond to ongoing flows and idle periods. Therefore, the number of ongoing flows (i.e., active users) in the network is not necessarily equal to the number of sessions in the network, due to "off" periods. We denote the number of ongoing PU flows and ongoing SU flows by $x_{1} \geq 0$ and $x_{2} \geq 0$ respectively and the total number of ongoing flows in the network by $x$, i.e $x=x_{1}+x_{2}$. Then, the network is saturated and users are forced to reduce their bit rate if $x>C / R$. We denote the critical number of ongoing flows at which the network starts to be saturated by $x_{c}=\left\lfloor\frac{C}{R}\right\rfloor$. In addition, we assume that the total number of ongoing flows is bounded by an arbitrarily large, but finite, number $M$. This network will be referred as a processor sharing network.

Next, we define the bandwidth allocation scheme used in this processor sharing network. We define the capacity allocated to PU flows and SU flows when there are $x$ ongoing flows in the network as follows:

$$
\phi_{i}(x)= \begin{cases}x_{i} R & \text { if } 0 \leq x<x_{c} \\ \frac{x_{i} C}{x} & \text { if } x_{c} \leq x \leq M,\end{cases}
$$

for $i=1,2$. We refer this processor sharing network as complete processor sharing. The bandwidth allocations are chosen such that they satisfy the balance property. A formal definition of the balance property is presented in Section 4.1.

When a PU enters the network it brings a reward $r_{1}$. However, the reward it brings decreases if its admittance causes any user to decrease its bit rate. This happens when the demand exceeds the capacity, i.e., $x>x_{c}$. We assume that there is an associated penalty for this event. We denote $0 \leq f_{1}(x) \leq r_{1}$ the penalty associated with the arrival of $\mathrm{PU}$ when the network is saturated or when its arrival causes the network to saturate. Similarly, if an SU is accepted to the network it brings a reward $r_{2}$. If an $\mathrm{SU}$ is admitted when the network is saturated or if it causes the network to saturate, the reward it brings decreases by an amount of $0 \leq f_{2}(x) \leq r_{2}$. We make the following assumptions on the penalty functions:

Assumption 1. $f_{1}(x)$ is convex and non-decreasing function in $x$, i.e. for $0 \leq x \leq M$

$$
\begin{aligned}
& f_{1}(x)-f_{1}(x+1) \geq f_{1}(x+1)-f_{1}(x+2), \\
& f_{1}(x)-f_{1}(x+1) \leq 0 .
\end{aligned}
$$

Assumption 2. $f_{2}(x)$ is non-decreasing function in $x$,

$$
\begin{aligned}
& \text { i.e. for } 0 \leq x \leq M \\
& \qquad f_{2}(x)-f_{2}(x+1) \leq 0 .
\end{aligned}
$$

We note that as a network becomes saturated and the load increases, then flows need to further reduce their bit rate. This result justifies the assumption that the penalty functions should be non-decreasing in the total number of ongoing flows $x$. The convexity assumption on $f_{1}(x)$ is needed for the derivation of the optimal admission control policies.

\section{ADMISSION CONTROL}

In this section we investigate an optimal insensitive occupancybased admission control policy on SUs that maximizes the average profit of a service provider under a general data traffic model. Occupancy-based admission control policies depend only on the number of ongoing flows in the network. The restriction to occupancy-based polices is not unrealistic, since it is difficult to track the amount of time each ongoing flow and session has spent in the network and predict the amount of time they will further stay. Furthermore, results in [20] indicate that the optimal occupancy-based admission control policy usually performs close to the optimal general admission control policy.

We assume that a service provider applies admission control on SUs only and PUs are always admitted unless the system is full. The possible controls on an arriving SU are acceptance or rejection. An occupancy-based admission control policy on SUs makes a decision based on the number of ongoing flows in the network, denoted $x$ (i.e., $x=x_{1}+x_{2}$ ). Therefore, the state space which defines the allowable values of state $x$ can be formulated as:

$$
\begin{aligned}
S=\{ & x \mid x=x_{1}+x_{2}, \phi_{1}(x) \leq x_{1} R, \phi_{2}(x) \leq x_{2} R, \\
& \left.\phi_{1}(x)+\phi_{2}(x) \leq C, 0 \leq x \leq M\right\} .
\end{aligned}
$$

We define a subspace $S_{c} \subset S$ that includes all the states for which the total bandwidth demand $\left(x_{1}+x_{2}\right) R$ exceeds the total capacity $C$, such that flows are forced to reduce their rate: $S_{c}=\left\{x \mid x>x_{c}, x \in S\right\}$. We refer to the states belonging to $S_{c}$ as "congestion states" since in these states elastic flows reduce their rates to avoid congestion.

At any state $x \in S \backslash M$, the control $u(x) \in U$ takes the value 0 or 1 , which corresponds to rejecting or accepting an $\mathrm{SU}$, respectively. We define an occupancy-based admission control policy $p=[u(0), u(1), \ldots, u(M-1)]$ which is a collection of controls $u(x) \in U$ for all states $x \in S \backslash M$. We denote the set of states in which an SU is admitted by $S_{a}$.

Next, we formulate the average profit function. The profit function consists of the following components: the rewards (including penalties) collected from the arrivals of PU sessions and admission of SU sessions. Then, the average profit rate, under a given policy $p$, is given as follows:

$$
\bar{V}_{p}=\sum_{x=0}^{M-1}\left(r_{1}-f_{1}(x)\right) \lambda_{1} \pi_{p}(x)+\sum_{x \in S_{a}}\left(r_{2}-f_{2}(x)\right) \lambda_{2} \pi_{p}(x),
$$

where $\pi_{p}(x)$ is the steady state probability of finding $x$ ongoing flows in the network. Due to the Poisson Arrivals See Time Averages (PASTA) property, the probability that a session arrival finds $x$ ongoing flows in the network is also $\pi_{p}(x)$. The first and second terms in (5) represent the average profit collected from PUs and accepted SUs respectively. 
Given the profit function, the objective function can be mathematically defined as follows. We are looking for the optimal admission control policy $p^{*}=\left[u^{*}(0), u^{*}(1), \ldots, u^{*}(M-\right.$ $1)$ ] that maximizes the average profit rate $\bar{V}_{p}$, i.e.,

$$
p^{*}=\underset{p}{\operatorname{argmax}}\left(\bar{V}_{p}\right) \text {. }
$$

The optimal profit under policy $p^{*}$ is denoted by $\bar{V}$, i.e.,

$$
\bar{V}=\bar{V}_{p^{*}} .
$$

\subsection{Insensitivity Property}

In this section we show that under a balanced bandwidth allocation scheme and session arrivals following a Poisson process, the steady state distribution of the number of ongoing flows in the network is insensitive to the traffic characteristics except through the traffic intensities. For this purpose, we show that the bandwidth allocation given by (1) is a sufficient condition for insensitivity.

Theorem 2 of [7] shows that partial reversibility of a network is a necessary and sufficient condition for the insensitivity property to hold. Moreover, [7] states that the reversibility of the arrival and service processes of a network is a sufficient condition for partial reversibility. It is also argued that the arrival process is reversible if and only if the arrival rates are balanced, and similarly the service process is reversible if and only if the service rates are balanced. Balanced arrival and service rates are defined as follows for a queue with multiple classes of users:

Definition 1. Consider $K$ class of users. Let define the state vector $\vec{x}=\left(x_{1}, x_{2}, \ldots, x_{K}\right)$, where $x_{i}$ corresponds to the number of ongoing flows of class $i$, with $i \in 1,2, \ldots, K$. The state-dependent arrival rates $\lambda(\vec{x})$ and service rates $\psi(\vec{x})$ at state $\vec{x}$ are balanced if and only if there exist positive functions $\Lambda(\vec{x})$ (with $\Lambda(0)=1$ ) and $\Psi(\vec{x})$, such that the arrival and service rates satisfy the following conditions:

$$
\begin{aligned}
\lambda(\vec{x}) & =\frac{\Lambda\left(\vec{x}+\overrightarrow{e_{i}}\right)}{\Lambda(\vec{x})}, \\
\psi(\vec{x}) & =\frac{\Psi\left(\vec{x}-\overrightarrow{e_{i}}\right)}{\Psi(\vec{x})},
\end{aligned}
$$

where $\overrightarrow{e_{i}}$ is a vector whose $i^{t h}$ entry is 1 and all other entries are 0 , and $x_{i}>0$ in Eq. (7).

We next show that for our network the bandwidth allocation given by (1) is a sufficient condition for partial reversibility. Hence, steady state distribution of the number of flows is insensitive to the traffic distributions.

THEOREM 1. For any admission control policy, the steady state probability distribution of the number of ongoing flows in the network is insensitive to the flow arrival and flow size distributions except through their means, provided that $P U$ and SU session arrivals follow independent Poisson processes and the bandwidth allocation satisfies (1).

Proof. We consider a network model, where PU and SU flows arrive according to independent Poisson processes, with rate $\lambda_{1}$ and $\lambda_{2}$ respectively. We assume that both PU and SU flows have a peak rate $R$ and exponentially distributed size with mean $\mu^{-1}$. We refer to this network model as the "Poisson flow arrival" model. We recall that a session is defined by consecutive "on" and "off" periods, where an "on" period corresponds to transmission of a flow. Then, the Poisson flow arrival model can be viewed as a special case of the Poisson session arrival model where each session consists of a single "on" period that is exponentially distributed.

Due to the homogeneous traffic assumption, PUs and SUs are indistinguishable once in the network. This assumption allows us to view the PU and SU flows as belonging to the same class with the following arrival rates

$$
\lambda_{p}(x)= \begin{cases}\lambda_{1}+\lambda_{2} & \text { if } u(x)=1, \\ \lambda_{1} & \text { if } u(x)=0\end{cases}
$$

where $\lambda_{p}(x)$ is the flow arrival rate under an admission control policy $p=[u(0), u(1), \ldots, u(M-1)]$ when there are $x \leq M-1$ flows in the network. When the bandwidth allocations of flows are defined as in (1), the total bandwidth allocated for PU and SU flows at state $x$ becomes:

$$
\phi(x)= \begin{cases}x R & \text { if } 0 \leq x<x_{c}, \\ C & \text { if } x_{c} \leq x \leq M .\end{cases}
$$

Then the service rates are $\psi(x)=\mu \phi(x)$ for all $x \in S$. For a single-class network, the balance functions exist for any admission control policy $p$ and are given as follows: $\Lambda(x)=$ $\prod_{i=1}^{x} \lambda_{p}(i-1)$ and $\Psi(x)=\left(\prod_{i=0}^{x} \psi(i)\right)^{-1}$.

By Definition 1, the existence of positive functions $\Lambda(x)$ and $\Psi(x)$, is a sufficient condition for balanced arrival and service rates. Thus, both arrival and service processes are reversible [7]. Therefore, when bandwidth allocations satisfy (1), the network is partially reversible [7]. Then, by Theorem 2 of [7] the steady state distribution of the number of flows in the network is insensitive to traffic characteristics, except through traffic means, provided that PU and SU sessions arrive according to independent Poisson processes.

Note that, from the outset, it is not obvious that the conditions of Definition 1 hold for our problem since PUs and SUs represent two different classes of users. However, we exploit the facts that PUs and SUs have identical service statistics and that the results of [7] on partial reversibility hold for state-dependent exogenous Poisson arrival processes. In this manner, we reduce the problem at hand into a single dimensional one (i.e., as if there were a single class of users). This result holds for any given admission control policy $p$ on SUs (including the optimal policy).

\subsection{Characterizing the Optimal Admission Con- trol Policy}

We next characterize the optimal occupancy-based admission control policy of SUs that maximizes the average profit rate. The insensitivity property guarantees that the steady state probabilities of the number of ongoing flows do not depend on the number of flows generated in the network and the distribution of the flow sizes. Therefore, we analyze the network under the Poisson flow arrival model. Under this model the evolution of the number of ongoing flows in the network can be modeled as a Markov process. Remarkably, the general optimal admission control policy for the Poisson flow arrival model is the same as the optimal occupancybased policy for the Poisson session arrival model.

\subsubsection{Dynamic Programming Formulation}


We formulate the profit maximization problem as a discounted discrete-time, finite-horizon stochastic dynamic programming problem. We apply standard techniques to generalize the results obtained for total discounted profit to analyze the infinite-horizon average profit case and characterize its properties. We note that the structural properties of the discrete-time, finite-horizon problem are the same as the continuous-time, infinite-horizon problem since the state space and control space are finite [6].

We consider a discount factor $\beta>0$ such that the profit earned at time $t$ is scaled by $\exp (-\beta t)$ [6] (page 254). This problem is a $\beta$-discounted, continuous-time, infinite-horizon Markov decision process (note that applying a discount factor to a process with an exponential rate $\beta$ is equivalent to terminating the process at an exponential rate $\beta$ [24]). Next, we use uniformization as described in [6] (page 254) to obtain the discrete-time equivalent of the continuous-time process. We normalize the transition rates and profit rate functions with a rate $v$, which is a transition rate that is no less than the maximum possible rate out of any state $x \in S$ under any control $u(x)$. Without loss of generality, we set the maximum possible rate out of any state to 1 , i.e., $v=\lambda_{1}+\lambda_{2}+C \mu+\beta=1$. Then, by following the techniques given in [6], the $\beta$-discounted continuous-time, infinite-horizon Markov decision process is converted into an $\alpha$-discounted discrete-time, infinite-horizon Markov decision process, where $\alpha=v /(\beta+v)$ and $0<\alpha<1$.

Next, we convert the infinite-horizon problem into a finitehorizon for ease of analysis of the properties of the profit function. Let $V_{n}^{\alpha}(x)$ be the optimal total expected $\alpha$-discounted profit function for the finite horizon, discrete-time, $n$ stage problem starting from state $x$. Then, Bellman's equations yielding the optimal policy for the finite-horizon, discounted problem can be written as:

- For $n=0, x \in S: V_{0}^{\alpha}(x)=0$

- For $n \geq 1,0 \leq x \leq x_{c}$ :

$$
\begin{aligned}
V_{n}^{\alpha}(x) & =\alpha \lambda_{1}\left(V_{n-1}^{\alpha}(x+1)+r_{1}-f_{1}(x)\right) \\
& +\alpha \lambda_{2} \max \left\{V_{n-1}^{\alpha}(x), V_{n-1}^{\alpha}(x+1)+r_{2}-f_{2}(x)\right\} \\
& +\alpha x R \mu V_{n-1}^{\alpha}(x-1)+\alpha(C-x R) \mu V_{n-1}^{\alpha}(x)
\end{aligned}
$$

- For $n \geq 1, x_{c}<x<M$ :

$$
\begin{aligned}
V_{n}^{\alpha}(x) & =\alpha \lambda_{1}\left(V_{n-1}^{\alpha}(x+1)+r_{1}-f_{1}(x)\right) \\
& +\alpha \lambda_{2} \max \left\{V_{n-1}^{\alpha}(x), V_{n-1}^{\alpha}(x+1)+r_{2}-f_{2}(x)\right\} \\
& +\alpha C \mu V_{n-1}^{\alpha}(x-1) .
\end{aligned}
$$

and $V_{n}^{\alpha}(M)=\alpha\left(\lambda_{1}+\lambda_{2}\right) V_{n-1}^{\alpha}(M)+\alpha C \mu V_{n-1}^{\alpha}(M-1)$. The boundary conditions are set as $V_{n}(-1)=V_{n}(0)$.

\subsubsection{Properties of the Optimal Admission Control Policy}

In this section we analyze the properties of the optimal admission control policy. First, one can show by induction on $n$ that $V_{n}^{\alpha}(x)$ satisfies the following concavity property.

Lemma 1. Under Assumptions 1 and 2 on the penalty functions, $V_{n}^{\alpha}(x)$ is concave in $x$ for all $x \in S$.

The proof appears in our technical report [15]. We return to the discrete-time, infinite-horizon average-profit problem. Given bounded profits and a compact control space, the infinite horizon discounted value function $V^{\alpha}(x)=\lim _{n \rightarrow \infty} V_{n}^{\alpha}$ exists [6], and it is concave.
For finite state and control spaces, it can be shown that, as $\alpha$ goes to 1 (i.e., as $\beta$ goes to 0 ), the optimal profit for the discounted problem converges (under proper scaling) to the optimal profit for the average problem, that is, $\bar{V}=\lim _{\alpha \rightarrow 1}(1-\alpha) V^{\alpha}(x)$ (the limit is independent of the initial state $x$ ), see [6] (page 194). These facts allow us to continue our analysis on the optimal admission control policy by focusing on the infinite-horizon average-profit problem.

For any $x \in S$, let $h(x)=\lim _{\alpha \rightarrow 1}\left(V^{\alpha}(x)-V^{\alpha}(0)\right)$. Then, Bellman's equations which yield the optimal admission control policy for the average profit problem can be written as: - For $0 \leq x \leq x_{c}$ :

$$
\begin{aligned}
\bar{V}+h(x) & =\frac{\lambda_{1}}{v}\left(h(x+1)+r_{1}-f_{1}(x)\right) \\
& +\frac{\lambda_{2}}{v} \max \left\{h(x), h(x+1)+r_{2}-f_{2}(x)\right\} \\
& +\frac{x R \mu}{v} h(x-1)+\frac{(C-x R) \mu}{v} h(x)
\end{aligned}
$$

- For $x_{c}<x<M$ :

$$
\begin{aligned}
\bar{V}+h(x) & =\frac{\lambda_{1}}{v}\left(h(x+1)+r_{1}-f_{1}(x)\right) \\
& +\frac{\lambda_{2}}{v} \max \left\{h(x), h(x+1)+r_{2}-f_{2}(x)\right\} \\
& +\frac{C \mu}{v} h(x-1)
\end{aligned}
$$

and $\bar{V}+h(M)=\frac{\lambda_{1}+\lambda_{2}}{v} h(x)+\frac{C \mu}{v} h(M-1)$. As before, we set $v$ to the maximum possible rate out of any state, i.e. $v=\lambda_{1}+\lambda_{2}+C \mu$.

By definition $h(x)$ inherits the properties of $V_{n}^{\alpha}(x)$. So, it is monotonically non-increasing and concave in $x$ for $x \in S$.

THEOREM 2. The optimal admission control policy $p^{*}$ of SUs is a threshold type policy, which depends only on the total number of ongoing flows in the network.

Proof. We first note that $h(x)-h(x+1)) \leq h(x+1)-$ $h(x+2)$ by concavity of $h(x)$. Thus, $h(x)-h(x+1)$ is monotonically non-decreasing in $x$ for all $x \in S$. From (11) and (12), we observe that an arriving SU is accepted if $h(x) \leq h(x+1)+r_{2}-f_{2}(x)$ and rejected otherwise. Define $x^{*}=\min \left\{x \mid h(x) \geq h(x+1)+r_{2}-f_{2}(x)\right\}$. For any $x>x^{*}$ such that $x \in S, h(x)>h(x+1)+r_{2}-f_{2}(x)$ holds by concavity of $h(x)$. Then for any $x>x^{*}$ the optimal decision is to reject an arriving $\mathrm{SU}$ and for any $x \leq x^{*}$ the optimal decision is to accept it. Thus, there exists a thresholds $x^{*}$ such that the optimal admission control policy of SU $p^{*}(x)$ in state $x$ can be written as:

$$
p^{*}(x)=\left\{\begin{array}{lll}
1(\text { accept }) & \text { if } x \leq x^{*}, & x \in S \\
0(\text { reject }) & \text { if } x>x^{*}, & x \in S
\end{array}\right.
$$

\subsection{Numerical Results}

In this section we provide numerical results. Throughout this section, we assume that an arriving PU brings a reward $r_{1}=10$ units and an accepted SU brings a reward $r_{2}=2$ units. We consider state dependent penalty functions such that it is guaranteed that $0 \leq f_{1}(x) \leq r_{1}$ and $0 \leq f_{2}(x) \leq r_{2}$ for all states $x \in S$. We also assume that the penalty on an arriving PU to a "congestion state" is not higher than the penalty on an accepted SU to a "congestion state", i.e. 
$f_{1}(x) \leq f_{2}(x)$ for all $x \in S$. Thus, the expected profit from an arriving $\mathrm{PU}$ at a given state is always higher than the expected profit an accepted SU would bring at the same state. These assumptions guarantee that PUs have higher priority than SUs.

First, as a sanity check, we confirm numerically the optimality of the threshold policy for different data traffic parameters by using policy iteration on the $\alpha$-discounted profit problem. For all examples, we consider a discount factor $\alpha=1-10^{-9}$ and we use the following penalty functions $f_{1}(x)$ and $f_{2}(x)$ (referred to as set 1): $f_{1}(x)=r_{2}\left(\frac{x-x_{c}}{M-x_{c}}\right)^{2}$ and $f_{2}(x)=r_{2}\left(\frac{x-x_{c}}{M-x_{c}}\right)$ if $x_{c}<x \leq M$; there is no penalty otherwise. The results obtained under different sample sets verify that the optimal policy is of threshold type. We also verify that the the maximum average profit obtained for the $\alpha$-discounted profit problem as $\alpha$ tends to 1 is the same as the results obtained for the average-profit problem.

Next, we present an alternative method which improves the running time performance of finding the optimal policy and the corresponding optimal average profit, given that the optimal occupancy-based policy is of threshold type. We find the optimal threshold and corresponding average profit by solving (5) for every possible threshold value and doing a linear search among the corresponding average profits to obtain the optimal threshold that gives the maximum average profit. Linear search on (5) yields the same optimal average profit as the policy iteration. To provide an insight on the advantage of using linear search over policy iteration, we consider the following example: For a network with the following parameters: $\lambda_{1}=150, \lambda_{2}=50, \mu=1, C=200$, $R=1, M=3000$, and the penalty functions as given by set 1. We compare the running times of linear search and policy iteration on a Dell x64-based PC with Intel Core i7-2600 $\mathrm{CPU}$ at $3.40 \mathrm{GHz}$ and $8 \mathrm{~GB}$ RAM. We find that the average running time of policy iteration is $3.8 \mathrm{sec}$ whereas linear search is $0.75 \mathrm{sec}$. Therefore, for practical purposes linear search method can be used to obtain the optimal threshold and expected profit. Our results also validate that linear search and policy iteration yield the same optimal threshold and corresponding average profit.

We next analyze the effect of the SU arrival rate $\lambda_{2}$ on the optimal threshold and the corresponding average profit for two different sets of penalty functions. We consider a network model where $\lambda_{1}=10, \mu=1, C=20$ units, $R=1$ unit, $M=100$ and analyze the change in optimal threshold and the corresponding average profit as $\lambda_{2}$ varies in the range $[5,10]$.

For the first set of penalty functions, we assume that the penalty functions $f_{1}(x)$ and $f_{2}(x)$ are defined as in set 1 . For the second set, we assume that both penalty functions $f_{1}(x)$ and $f_{2}(x)$ are a constant defined as: $f_{1}(x)=f_{2}(x)=1$ if $x_{c}<x \leq M$ and there is no penalty otherwise. We refer to these penalty functions as set 2 .

Figure 1 and Figure 2 show the dependence of the optimal threshold and the corresponding average profit upon $\lambda_{2}$. We observe that for both set 1 and set 2 , the optimal threshold decreases with increasing $\lambda_{2}$ as the network saturates faster and the reward gained from PUs and SUs starts decreasing due to the non-zero penalty functions. We note that the optimal threshold and the corresponding average profit values obtained under set 1 are higher than those obtained under set 2. This is due to the lower penalty values of set 1 for most of the states. In addition, the gap between the optimal average profits obtained under set 1 and set 2 increases with $\lambda_{2}$ since under set 1 the optimal threshold decreases significantly as $\lambda_{2}$ increases. We note that, for both models, the optimal average profit increases sub-linearly with $\lambda_{2}$ since the optimal threshold decreases with $\lambda_{2}$.

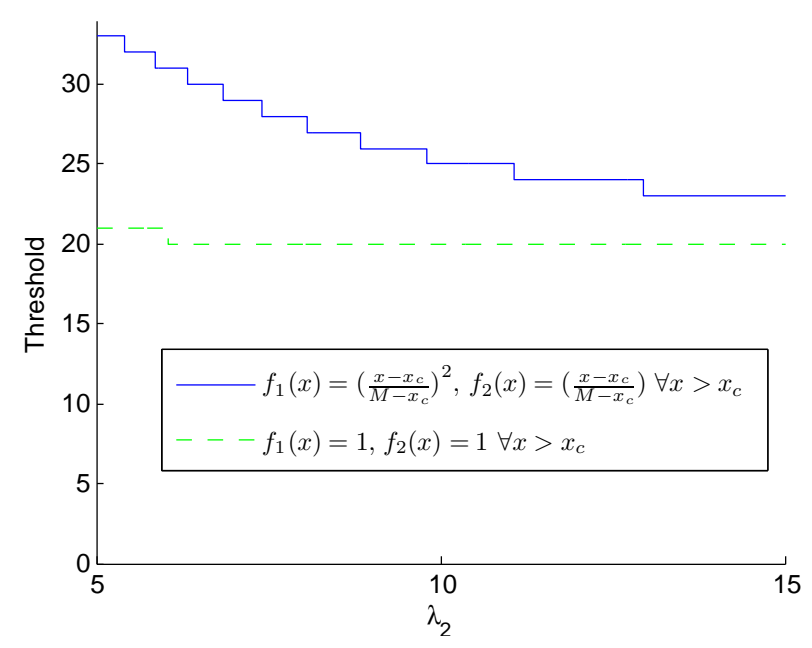

Figure 1: Optimal threshold versus SU arrival rate where the data traffic parameters are as follows: $\lambda_{1}=10, \mu=1, C=20, R=1, M=100, r_{1}=10$ and $r_{2}=2$. The threshold decreases with increasing secondary demand.

\section{PROFITABILITY ANALYSIS}

So far, we have assumed that the arrival rate of SUs $\lambda_{2}$ is known and given. In practice, however, $\lambda_{2}$ is a function of the secondary price $r_{2}$. This function, referred to as the demand function, is not always easy to characterize. In this section, we analyze the conditions under which secondary spectrum provisioning generates additional profit, irrespective of the shape of the secondary demand function.

We investigate profitable prices under the Poisson flow arrival model. By the insensitivity property, the profitability conditions obtained for the Poisson flow arrival model hold for the original Poisson session arrival model.

We first consider a system where all SUs are rejected, thus, the system only serves PUs. We define this regime as the lockout policy. Under the lockout policy the total number of ongoing flows in the network, denoted by $x$, evolves as a Markov process and the steady state probabilities can be obtained by solving the detailed balance equations.

The steady state probabilities $\pi_{x}$ to find the network in state $x$ under the lockout policy is as follows:

$$
\pi_{x}^{L O}= \begin{cases}\frac{\rho_{1}^{x}}{R^{x} x !} G^{L O} & \text { if } 0 \leq x \leq x_{c} \\ \frac{\rho_{1}{ }^{x}}{C^{x-x_{c} R^{x} x_{c} !}} G^{L O} & \text { if } x_{c}<x \leq M\end{cases}
$$

where the normalization constant $G^{L O}$ is

$$
G^{L O}=\left(\sum_{j=0}^{x_{c}} \frac{\rho_{1}^{j}}{R^{j} j !}+\sum_{j=x_{c}+1}^{M} \frac{\rho_{1}^{j}}{C^{j-x_{c}} R^{x_{c}} x_{c} !}\right)^{-1} .
$$




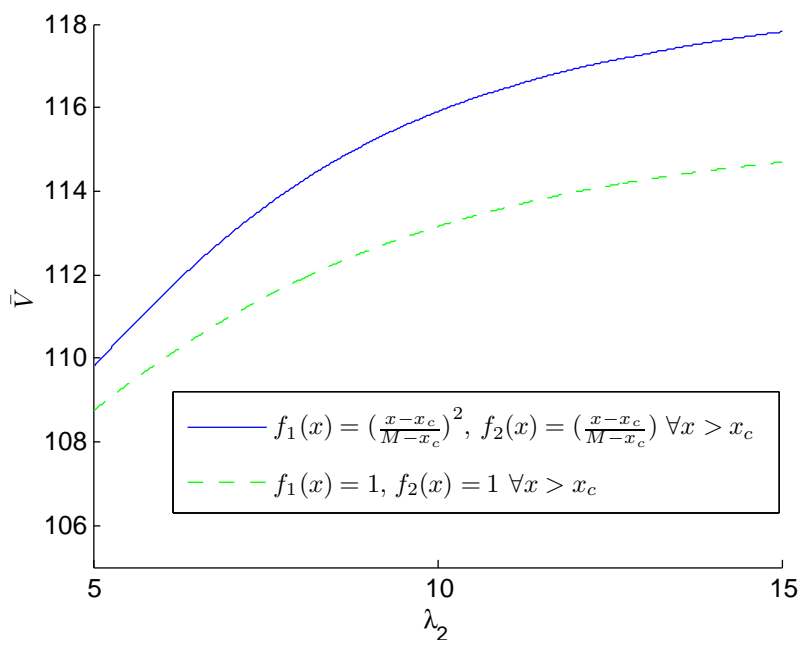

Figure 2: Optimal average profit versus SU arrival rate where the data traffic parameters are as follows: $\lambda_{1}=10, \mu=1, C=20, R=1, M=100, r_{1}=10$ and $r_{2}=2$. The average profit increases sublinearly with increasing secondary demand. is:

Then the average profit collected from the lockout policy

$$
\bar{V}^{L O}=\lambda_{1} \sum_{x=0}^{M-1}\left(r_{1}-f_{1}(x)\right) \pi_{x}^{L O}
$$

An admission control policy of SU is profitable for a given price $r_{2}$ and secondary demand $\lambda_{2}$ if the average profit collected by admitting SUs under this policy is larger than the average profit obtained by the lockout policy. Then the profitability region can be defined as the set of $\left(r_{2}, \lambda_{2}\right)$ pairs that are profitable under this policy.

Next, for each secondary demand $\lambda_{2}$ we search for a secondary price $r_{2}^{*}$ such that there exists no profitable admission control policy with a secondary price lower than $r_{2}^{*}$. To accomplish this, we search for the conditions when it is profitable to admit an SU request.

We formulate the problem as an infinite horizon average profit optimization problem to analyze the optimal conditions for admitting SU requests. As before, let $\bar{V}$ denote the discrete-time, infinite-horizon average-profit. Then, Bellman's equations for the discrete-time, infinite-horizon average-profit problem that yields the optimal admission control policy of SUs is given by (11) and (12).

We use policy improvement arguments to find the secondary prices that are profitable for a given secondary demand $\lambda_{2}$. We start with the lockout policy and search for the secondary price $r_{2}$ that improves the average profit. Under the lockout policy, Bellman's equations are:

$$
\begin{aligned}
\bar{V}^{L O}+h^{L O}(x) & =\frac{\lambda_{1}}{v}\left(h^{L O}(x+1)+r_{1}-f_{1}(x)\right)+\frac{\lambda_{2}}{v} h^{L O}(x) \\
& +\max \left(\frac{x R \mu}{v}, \frac{C \mu}{v}\right) h^{L O}(x-1) \\
& +\max \left(\frac{(C-x R) \mu}{v}, 0\right) h^{L O}(x) .
\end{aligned}
$$

and

$$
\bar{V}^{L O}+h^{L O}(M)=\frac{\lambda_{1}+\lambda_{2}}{v} h^{L O}(x)+\frac{C \mu}{v} h^{L O}(M-1) .
$$

By policy improvement, an $\mathrm{SU}$ is admitted at state $x$ only if $\max \left\{h^{L O}(x), h^{L O}(x+1)+r_{2}-f_{2}(x)\right\}=h^{L O}(x+1)+$ $r_{2}-f_{2}(x)$. Thus, to admit an SU request at state $x$ the secondary price $r_{2}$ needs to satisfy the following condition:

$$
r_{2}>h^{L O}(x)-h^{L O}(x+1)+f_{2}(x) .
$$

The above condition on $r_{2}$ can also be deduced intuitively by realizing that $h^{L O}(x)-h^{L O}(x+1)$ gives the difference in the profit rate of the lockout policy for starting at state $x$ instead of state $x+1$. Therefore, $r_{2}$ needs to exceeds $h^{L O}(x)-h^{L O}(x+1)+f_{2}(x)$ which represents the cost of adding an SU when the network is in state $x$.

A profitable price should satisfy this condition independent of the system state. We define this price as the breakeven price denoted by $r_{2}^{*} \geq 0$, where.

$$
r_{2}^{*}=\min _{x=0,1,2, \ldots, M} h^{L O}(x)-h^{L O}(x+1)+f_{2}(x) .
$$

By definition, for a given secondary demand $\lambda_{2}$, there exists no profitable price that is lower than $r_{2}^{*}$. In addition, we note that $r_{2}^{*}$ is independent of the secondary demand $\lambda_{2}$. Therefore, for any secondary price $r_{2} \geq r_{2}^{*}$ there exists a profitable admission control policy, irrespective of the shape of the secondary demand function.

The next theorem explicitly computes the break-even price and associated demand-insensitive profitable admission control policy.

THEOREM 3. The break-even price for admitting SUs to the network is

$$
r_{2}^{*}=r_{1} \pi_{M}^{L O}+\sum_{x=x_{c}+1}^{M-1} f_{1}(x) \pi_{x}^{L O} .
$$

The admission control policy, which is profitable for any secondary price $r_{2}$ that exceeds the break-even price $r_{2}^{*}$, admits secondary traffic when $x=0$. Furthermore, this admission control policy is robust to variations in the secondary demand function $\lambda_{2}$.

ProOF. We apply policy improvement on the lockout policy to find the break-even price, using a similar approach to $[2,3]$. To characterize the break-even price, we consider an admission control policy that provides secondary spectrum access to improve the lockout policy. We refer to this policy as the "sharing policy" since the profit is improved by sharing the network resources with SUs. We adopt a threshold type occupancy-based admission control policy since it has been shown in Section 4 to be optimal among all occupancy-based admission control policies. This policy suggests that an SU request is accepted if the total number of ongoing flows in the system is less than or equal to $T$. Thus, this policy guarantees that there can be at most $T+1$ ongoing SU flows in 
the network. Equation (17) shows that the break-even price $r_{2}^{*}$ is independent of the secondary demand $\lambda_{2}$. Therefore, we can set $\lambda_{2}=\infty$. Then, the total number of the ongoing flows in the network evolves as a Markov process.

By setting $\lambda_{2}=\infty$, it is guaranteed that there are always $T+1$ SUs in the network. Thus the number of ongoing flows in the network can only take values in the range $[T+1, M]$. The steady state probabilities $\pi_{x}$ to find the system in state $x$ under this policy is:

- For $T<x_{c}$

$\pi_{x}^{S P}= \begin{cases}0 & \text { if } 0 \leq x \leq T, \\ \frac{\rho_{1}{ }^{x}}{1_{x \leq x_{c}}\left(R^{x} x !\right)+1_{x>x_{c}}\left(C^{x-x_{c}} R^{\left.x_{c} x_{c} !\right)}\right.} G^{S P}, & \text { if } T<x<M,\end{cases}$

where $1_{\{.\}}$is the indicator function and the normalization constant $G^{S P}$ is

$$
G^{S P}=\left(\sum_{j=T+1}^{x_{c}} \frac{\rho_{1}^{j}}{R^{j} j !}+\sum_{j=x_{c}+1}^{M} \frac{\rho_{1}^{j}}{C^{j-x_{c}} R^{x_{c}} x_{c} !}\right)^{-1} .
$$

- For $T \geq x_{c}$

$$
\pi_{x}^{S P}= \begin{cases}0 & \text { if } 0 \leq x \leq T, \\ \frac{\rho_{1}{ }^{x}}{C^{x-x_{c} R^{x} c x_{c} !}} G^{S P} & \text { if } T<x<M,\end{cases}
$$

where the normalization constant $G^{S P}$ is

$$
G^{S P}=\left(\sum_{j=T+1}^{M} \frac{\rho_{1}^{j}}{C^{j-x_{c}} R^{x_{c}} x_{c} !}\right)^{-1}
$$

Then the average profit collected from this policy is:

$$
\bar{V}^{S P}=\lambda_{2} \sum_{x=0}^{T}\left(r_{2}-f_{2}(x)\right) \pi_{x}^{S P}+\lambda_{1} \sum_{x=T+1}^{M-1}\left(r_{1}-f_{1}(x)\right) \pi_{x}^{S P} .
$$

The first and second terms correspond to the profit collected from SUs and PUs respectively. With the $\lambda_{2}=\infty$ assumption, the profit collected from SUs becomes $\lambda_{2}\left(r_{2}-\right.$ $\left.f_{2}(T)\right) \pi_{T}^{S P}=\infty * 0$. We use the balance equations at state $T$ to obtain the average profit collected from SUs, which is:

$$
\lambda_{2} \pi_{T}^{S P}= \begin{cases}(T+1) R \mu \pi_{T+1}^{S P} & \text { if } T<x_{c} \\ C \mu \pi_{T+1}^{S P} & \text { if } T \geq x_{c}\end{cases}
$$

The policy improvement argument adopts the sharing policy if it is more profitable than the lockout policy. In other words service provider provides secondary spectrum access if the profit rate of the sharing policy exceeds the profit rate of the lockout policy, i.e.

$$
\bar{V}^{S P}>\bar{V}^{L O}
$$

We analyze the range of the profitable secondary prices as a function of $T$ and prove that the lowest is reached for $T=$ 0 . Since the profit rate under sharing policy $\bar{V}^{S P}$ changes depending on the relationship between $T$ and $x_{c}$, we analyze the profitable prices for $T<x_{c}$ and $T \geq x_{c}$ separately.

First, we investigate the profitable secondary prices when $T<x_{c}$. Under this assumption Eq. (22) becomes

$$
\begin{aligned}
& (T+1) R \mu\left(r_{2}-f_{2}(T)\right) \pi_{T+1}^{S P}+\lambda_{1} \sum_{x=T+1}^{M-1}\left(r_{1}-f_{1}(x)\right) \pi_{x}^{S P} \\
& >\lambda_{1} \sum_{x=0}^{M-1}\left(r_{1}-f_{1}(x)\right) \pi_{x}^{L O} .
\end{aligned}
$$

We are interested in finding the largest profitable region. We search for the optimum $T$ that minimizes $r_{2}$ satisfying Eq. (23). As defined in Section 3, the penalty functions $f_{1}(x)$ and $f_{2}(x)$ are introduced when the system is in a congestion state, therefore, $f_{2}(T)=0$ for $T<x_{c}$. Then, the minimum secondary price can be defined by combining the steady state probabilities given by (14) and (18) and regrouping the terms, as follows:

$r_{2}^{*}=\min _{T=0,1,2, \ldots, x_{c}-1}\left\{\left(\frac{R^{T+1}(T+1) !}{\rho_{1}^{T}(T+1) R}\right)\left(\frac{1}{G^{L O}}-\frac{1}{G^{S P}}\right) a^{\prime}\right\}$,

where $a^{\prime}=r_{1} \pi_{M}^{L O}+\sum_{x=x_{c}+1}^{M-1} f_{1}(x) \pi_{x}^{L O}$ is a constant independent of $T$.

By using induction we observe that (24) is increasing in $T$ for all $T \in\left[0, x_{c}-1\right]$.

Next, we analyze the second case where $T \geq x_{c}$. Under this condition (22) becomes

$$
\begin{aligned}
& C \mu\left(r_{2}-f_{2}(T)\right) \pi_{T+1}^{S P}+\lambda_{1} \sum_{x=T+1}^{M-1}\left(r_{1}-f_{1}(x)\right) \pi_{x}^{S P} \\
& >\lambda_{1} \sum_{x=0}^{M-1}\left(r_{1}-f_{1}(x)\right) \pi_{x}^{L O} .
\end{aligned}
$$

Similar to the previous case we search for the optimal $T$ that results in the minimum $r_{2}$. After rearranging the terms, (25) can be written as follows:

$$
\begin{aligned}
r_{2}^{*}=\min _{T=x_{c}, x_{c}+1,2, \ldots, M} & \left(f_{2}(T)-\frac{\rho_{1}}{C \pi_{T+1}^{S P}} \sum_{x=T+1}^{M-1}\left(r_{1}-f_{1}(x)\right) \pi_{x}^{S P}\right. \\
& \left.+\frac{\rho_{1}}{C \pi_{T+1}^{S P}} \sum_{x=0}^{M-1}\left(r_{1}-f_{1}(x)\right) \pi_{x}^{L O}\right) .
\end{aligned}
$$

We verify that each term of (26) is increasing in $T$ and conclude that the minimum is reached at $T=x_{c}$ in the range $\left[x_{c}, M-1\right]$.

Next, we investigate the optimal value of $T$ in the range $[0, M-1]$. We analyze the maximum secondary price obtained for $T<x_{c}$ and the minimum secondary price obtained for $T \geq x_{c}$ and show that $r_{2}^{*}$ is increasing in $T$ for all $T$ in the range $[0, M-1]$. We remind that both $(24)$ and (26) increase in $T$. Thus, the maximum secondary price for $T<x_{c}$ is obtained at $T=x_{c}-1$ and it is given by

$$
R_{2,1}^{*}=\frac{R^{x_{c}} x_{c} !}{\rho_{1}^{x_{c}-1} C}\left(\sum_{x=0}^{x_{c}-1} \frac{\rho_{1}^{x}}{R^{x} x !}\right)\left(r_{1} \pi_{M}^{L O}+\sum_{x=x_{c}+1}^{M-1} f_{1}(x) \pi_{x}^{L O}\right),
$$

whereas, the minimum secondary price for $T \geq x_{c}$ is obtained at $T=x_{c}$ and is given by

$$
R_{2,2}^{*}=\frac{R^{x_{c}} x_{c} !}{\rho_{1}^{x_{c}}}\left(\sum_{x=0}^{x_{c}} \frac{\rho_{1}^{x}}{R^{x} x !}\right)\left(r_{1} \pi_{M}^{L O}+\sum_{x=x_{c}+1}^{M-1} f_{1}(x) \pi_{x}^{L O}\right) .
$$

We rearrange the terms in Eq. (27), then

$$
R_{2,2}^{*}=\frac{C}{\rho_{1}} R_{2,1}^{*}+\left(r_{1} \pi_{M}^{L O}+\sum_{x=x_{c}+1}^{M-1} f_{1}(x) \pi_{x}^{L O}\right) .
$$

In Section 3 it is assumed that $\rho_{1}<C$. This assumption guarantees that $R_{2,2}^{*}>R_{2,1}^{*}$. Therefore, $r_{2}^{*}$ is increasing in 


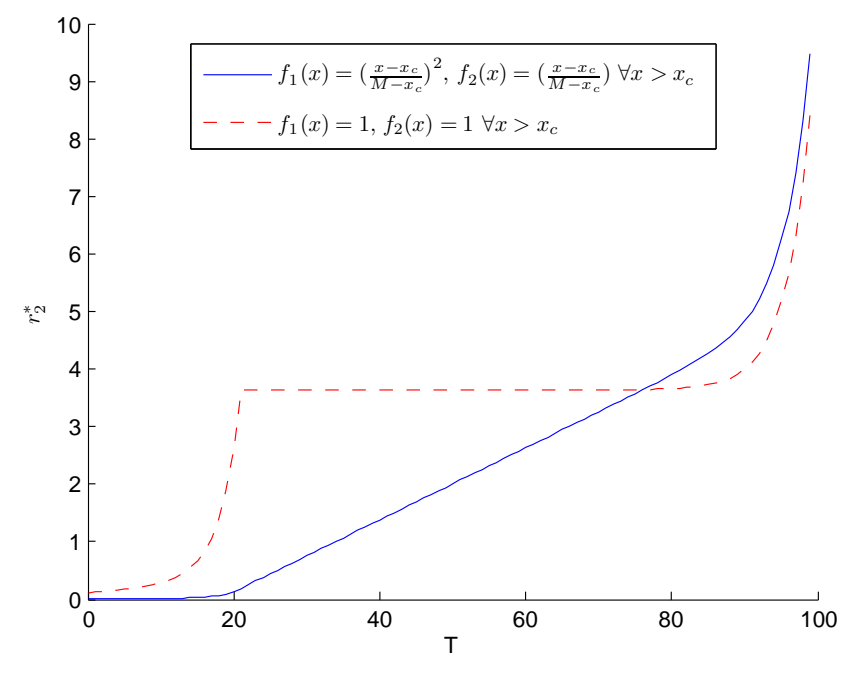

Figure 3: Break-even price $r_{2}^{*}$ vs optimal threshold $T$ on a network model with following parameters: $M=100, \lambda_{1}=15, \mu=1, C=20, R=1$ and $r_{1}=10$. Lowest profitable price is reached at $T=0$.

$T$ for all $T$ in the range $[0, M-1]$. We conclude that the minimum $r_{2}^{*}$ is reached at $T=0$, which is equal to

$$
R_{2}^{*}=r_{1} \pi_{M}^{L O}+\sum_{x=x_{c}+1}^{M-1} f_{1}(x) \pi_{x}^{L O} .
$$

As long as the secondary price $r_{2}$ is higher than the break even price, i.e. $r_{2}>r_{2}^{*}$, then the optimal occupancy-based admission control policy with threshold $T=0$ is always profitable, independent of the secondary demand $\lambda_{2}$. This policy achieves the largest profitability region.

We illustrate the optimal threshold that results in the largest profitable region under two penalty sets set 1 and set 2 as defined before. Figure 3 demonstrates that the breakeven price is reached at $T=0$ for both sets under a network model where $M=100, \lambda_{1}=15, \mu=1, C=20$ units, $R=1$ unit and $r_{1}=10$.

\section{CONCLUSIONS}

We studied the problem of admission control and pricing of secondary users in DSA networks. Our main contribution is in the derivation of results that are insensitive to traffic characteristics, except for the traffic intensity (average load), and to the relationship between price and demand. Thus, these results provide important guidelines for providers wishing to enter secondary spectrum markets.

Specifically, we first analyzed a two-class revenue maximization problem in a DSA processor sharing network with homogeneous, elastic data traffic. We showed that the value of an additional PU or SU flow is non-increasing and concave in the number of initial ongoing flows in the network. Using this property, we proved that the optimal occupancybased admission control policy on SUs is of threshold type and depends only on the total number of ongoing flows in the network. We demonstrated that once the optimality of threshold type policy is known, a simple linear search procedure outperforms policy iteration. We note that these results hold for general forms of penalty functions satisfying mild conditions (cf. Assumption 1). This leaves room for providers to optimize their system performance and profit, though the issue of how to properly set these functions remains open.

We next leveraged our results on the structure of optimal admission control policies to analyze profitability of secondary spectrum leasing under uncertain demand. We provided an analytical formula for the break-even price. We observed that the break-even price is smaller than the primary reward and corresponds to the opportunity cost of the lockout policy to start with one user in the network versus none. We proved that for any price above the break-even price it is always profitable to accept secondary users when no user is present in the network. Therefore, for any price above the break-even price profitability is guaranteed, regardless of the secondary demand function.

Remarkably, the optimal admission control policy and the profitability conditions obtained for general data traffic models and processor sharing networks resemble those obtained for inelastic streaming traffic in loss networks. Thus, we expect that results obtained in $[2,3,13,19,20]$ can be generalized to a much larger class of traffic and resource allocation models than considered therein.

\section{Acknowledgments}

This research was supported in part by NSF under grant CCF-0964652.

\section{REFERENCES}

[1] I. Akyildiz, W. Lee, M. Vuran, and S. Mohanty. NeXt generation/dynamic spectrum access/cognitive radio wireless networks: A survey. Computer Networks, 50(13):2127-2159, 2006.

[2] A. Al Daoud, M. Alanyali, and D. Starobinski. Profit-robust policies for dynamic sharing of radio spectrum. In Global Communications Conference (GLOBECOM), 2012 IEEE, pages 1186-1191, 2012.

[3] M. Alanyali, A. Al Daoud, and D. Starobinski. Profitability of dynamic spectrum provision for secondary use. In IEEE Symposium on New Frontiers in Dynamic Spectrum Access Networks (DySPAN), pages 136-145. IEEE, 2011.

[4] M. Arlitt and C. Williamson. Internet web servers: Workload characterization and performance implications. IEEE/ACM Transactions on Networking (ToN), 5(5):631-645, 1997.

[5] M. Becchi. From Poisson processes to self-similarity: a survey of network traffic models. http://www1.cse. wustl.edu/ jain/cse567-06/ftp/traffic_models1/, 2008.

[6] D. Bertsekas. Dynamic Programming and Optimal Control Vol 2. Athena Scientific, second edition, 2001.

[7] T. Bonald. Insensitive queueing models for communication networks. In Proceedings of the 1st International Conference on Performance Evaluation Methodologies and Tools, page 57. ACM, 2006. 
[8] T. Bonald and A. Proutiere. A queueing analysis of data networks. In Queueing Networks, pages 729-765. Springer, 2011.

[9] Cisco. Cisco visual networking index: Global mobile data traffic forecast update, 2012-2017. http://www.cisco.com/en/US/solutions/ collateral/ns341/ns525/ns537/ns705/ns827/ white_paper_c11-520862.pdf, 2012.

[10] FCC. Docket no 03-222 notice of proposed rule making and order, 2003.

[11] S. Floyd and V. Paxson. Difficulties in simulating the internet. IEEE/ACM Transactions on Networking (TON), 9(4):392-403, 2001.

[12] M. Jonckheere and J. Mairesse. Towards an Erlang formula for multiclass networks. Queueing Systems, 66(1):53-78, 2010.

[13] E. Kavurmacioglu, M. Alanyali, and D. Starobinski. Competition in secondary spectrum markets: Price war or market sharing? In IEEE Dynamic Spectrum Access Networks (DYSPAN) International Symposium on, pages 440-451. IEEE, 2012.

[14] H. Kim and K. G. Shin. Optimal admission and eviction control of secondary users at cognitive radio hotspots. In 6th Annual IEEE Communications Society Conference on Sensor, Mesh and Ad Hoc Communications and Networks (SECON), pages 1-9. IEEE, 2009.

[15] S. Kockan and D. Starobinski. Admission control and profitability analysis in dynamic spectrum access data networks. Technical Report 2013-IR-0005, CISE, Boston University, August 2013. Also available as http://www.bu.edu/phpbin/cise/download.php? publication_id=1257.

[16] A. Kortebi, S. Oueslati, and J. Roberts. Cross-protect: implicit service differentiation and admission control. In High Performance Switching and Routing, 2004. HPSR. 2004 Workshop on, pages 56-60, 2004.

[17] X. Li, U. Toseef, T. Weerawardane, W. Bigos, D. Dulas, C. Goerg, A. Timm-Giel, and A. Klug. Dimensioning of the LTE access transport network for elastic Internet traffic. In IEEE 6th International Conference on Wireless and Mobile Computing, Networking and Communications (WiMob), pages 346-354. IEEE, 2010.

[18] B. Miller. A queueing reward system with several customer classes. Management Science, 16:234-245, 1971.

[19] H. Mutlu, M. Alanyali, and D. Starobinski. Spot pricing of secondary spectrum access in wireless cellular networks. IEEE/ACM Transactions on Networking (TON), 17(6):1794-1804, 2009.

[20] H. Mutlu, M. Alanyali, and D. Starobinski. On-line pricing of secondary spectrum access with unknown demand function and call length distribution. In IEEE International Conference on Computer Communications (INFOCOM), pages 947-955, 2010.

[21] V. Pla, J. Virtamo, and J. Martínez-Bauset. Optimal robust policies for bandwidth allocation and admission control in wireless networks. Computer Networks, 52(17):3258-3272, 2008.

[22] R. Ramjee, D. Towsley, and R. Nagarajan. On optimal call admission control in cellular networks. Wireless
Networks, 3:29-41, 1996.

[23] A. Turhan, M. Alanyali, and D. Starobinski. Optimal admission control of secondary users in preemptive cognitive radio networks. In 10th International Symposium on Modeling and Optimization in Mobile, Ad Hoc and Wireless Networks (WiOpt), pages 138-144. IEEE, 2012.

[24] J. Walrand. An Introduction to Queueing Networks. Prentice Hall, 1988.

[25] F. Wang, J. Huang, and Y. Zhao. Delay sensitive communications over cognitive radio networks. IEEE Transactions on Wireless Communications, 11(4):1402-1411, 2012.

[26] C. Williamson. Internet traffic measurement. IEEE Internet Computing, 5(6):70-74, 2001. 